\title{
Computational analysis identifies invasion-associated genes in pituitary adenomas
}

\author{
CHANGJUN CAO ${ }^{*}$, WEI WANG ${ }^{*}$, CHAO MA and PUCHA JIANG \\ Department of Neurosurgery, Zhongnan Hospital of Wuhan University, Wuhan, Hubei 430071, P.R. China
}

Received June 16, 2014; Accepted March 4, 2015

DOI: $10.3892 / \mathrm{mmr} .2015 .3564$

\begin{abstract}
Pituitary adenomas are considered to be benign tumours. However, they can infiltrate surrounding tissues, which may cause a failure of complete removal during surgical resection. Thus far, no molecular biomarkers have been identified, which are able to reliably predict the behaviour of this type of tumour. In the present study, a list of differentially expressed genes in invasive pituitary adenomas was obtained using a computational bioinformatics analysis on the DNA microarray expression profiles. The gene expression datasets of a total of 16 samples were retrieved from the National Center for Biotechnology Information Gene Expression Omnibus database. The gene set enrichment analysis was later conducted on the significantly $($ FDR $<0.05)$ differentially expressed genes. A total of 194 genes were identified as differentially expressed. The pathway impact analysis revealed that cell adhesion molecules may be vital in the progression of pituitary adenoma invasion. A total of six genes, claudin 7 , contactin associated protein-like 2 , integrin $\alpha 6$, junctional adhesion molecule 3 , protein tyrosine phosphatase, receptor type $C$ and cadherin-associated protein $\alpha 1$ were identified as molecular biomarkers for pituitary adenoma invasion. The present study identified six novel molecular biomarkers, which may be used for diagnostic or therapeutic purposes. However, further experimental investigations are required to validate the present findings.
\end{abstract}

\section{Introduction}

Pituitary adenomas are common benign neoplasms, which may cause disorders of growth, reproductive function and cortisol production (1). Surgical resection is the treatment of choice for the majority of symptomatic pituitary adenomas. However, due to extensive local invasion, removal of the complete tumour

Correspondence to: Mr. Pucha Jiang, Department of Neurosurgery, Zhongnan Hospital of Wuhan University, 169 Donghu Road, Wuhan, Hubei 430071, P.R. China

E-mail: jiangpucha64@163.com

${ }^{*}$ Contributed equally

Key words: invasion, pituitary adenomas, microarray is difficult (2). Although not malignant, invasive pituitary adenomas are able to infiltrate surrounding tissues, including the dura mater, the cranial bone, or the sphenoid sinus (2). The invasions observed at the time of surgery account for up to $35 \%$ of pituitary adenomas (1). By contrast, the metastases to the pituitary are rare, accounting for $0.1-0.2 \%$ of pituitary tumours (2). Invasive pituitary adenomas remain clinically indistinguishable prior to identification of metastases. Thus, numerous attempts have been made to define molecular markers associated with invasive adenoma. For example, there is a significant association between the expression of the tumour protein p53 (TP53) gene and the behaviour of the pituitary tumour. The overexpression of TP53 was observed in $100 \%$ of pituitary carcinomas, $15 \%$ of invasive adenomas and $0 \%$ of non-invasive adenomas examined in a previous study (3). The fibroblast growth factor receptor 4 gene has been revealed to induce pituitary tumour invasion in in vivo animal models in association with reduced $\mathrm{N}$-cadherin expression (4). The gene expression of matrix metalloproteinase 9 was higher in invasive tumours compared with non-invasive pituitary tumours (5). Notably, no single marker has been identified to reliably predict the behaviour of the tumour thus far (6), and whether such a biomarker may be able to improve clinical management and the ultimate outcome remains to be elucidated.

DNA microarray technology has been developed to measure the expression of thousands of genes simultaneously in one single experiment. Over the past few years, a number of raw datasets from DNA microarray experiments have been deposited in public primary databases, such as the National Center for Biotechnology Information (NCBI) Gene Expression Omnibus (GEO) (7) and the European Molecular Biology Laboratory-European Bioinformatics Institute ArrayExpress (8). Therefore, researchers are able to reuse the resources by conducting comprehensive computational analysis. In the present study, the DNA microarray expression profiles of invasive pituitary adenomas were retrieved from the NCBI GEO database and invasion-associated genes were identified using a computational bioinformatics analysis pipeline. This gene signature provided novel diagnostic biomarkers and therapeutic targets for the treatment of invasive pituitary adenomas.

\section{Materials and methods}

DNA microarray expression datasets. The gene expression datasets of invasive and non-invasive pituitary adenomas were 
retrieved from a public functional genomics data repository, the NCBI GEO database. As shown in Table I, a total of 16 samples were analysed in the present study, eight non-invasive and eight invasive pituitary adenomas. The DNA microarray experiments were based on the GPL570 [HG-US133 Plus 2] Affymetrix Human Genome U133 Plus 2.0 Array platform (Affymetrix, Santa Clara, CA, USA), which included complete coverage of the Human Genome U133 Set plus 9,921 new probe sets representing $\sim 6,500$ additional genes for the analysis of $>47,000$ transcripts.

Data pre-processing. The intensity files with a CEL file extension suffix for each sample were loaded in $\mathrm{R}$ by the R/Bioconductor (http://www.bioconductor.org/) package, Affy (9), to create an AffyBatch object. Subsequently, the Bioconductor 'gcrma' package was used for background correction and normalization. This function added a step of adjusting the expression values based on the propensity of certain probes to undergo non-specific binding. The output of this process was an expression set object. The data sets were $\log 2$ transformed for gene expression comparison analysis.

Obtaining the ranked list of differentially expressed genes. To identify differentially expressed genes across multiple datasets, a non-parametric method was employed and implemented in the RankProd (10) package. RankProd is a statistically rigorous, but biologically intuitive algorithm, which has been demonstrated to be robust against noise in microarray data. This algorithm has also been revealed to have a higher sensitivity and specificity compared with other types of meta-analytic tools for microarrays.

Gene set enrichment analysis. To more thoroughly characterize sets of functionally related genes differentially expressed between non-invasive and invasive pituitary adenoma samples, Onto-Express (11) was used to classify genes according to the following Gene-Ontology (GO) categories: Biological process; cellular component; and molecular function. An impact analysis was used to identify the pathways affected by the differentially expressed genes in the invasive pituitary adenomas. The Kyoto Encyclopedia of Genes and Genomes (KEGG) pathway enrichment analysis was used to identify important pathways involved in invasive pituitary adenomas. The KEGG database is well-known for a comprehensive database focusing on various biochemistry pathways. This systems biology approach was implemented by the Web-based tool, Pathway-Express (11).

\section{Results}

Identification of genes differentially expressed under an invasive state. Although the microarray experiments were conducted by various groups (Table I), the gcrma function conducted background correction and normalization on the raw data during the pre-processing stage (Fig. 1). The RankProd function yielded a list of genes ranked by percentage of false positive prediction ( $\mathrm{pfp}$ ) values. In the current study, a total of 194 genes, including 55 upregulated and 139 downregulated genes, were identified when the threshold was set as $\mathrm{pfp}<0.05$ and $\log \mathrm{FCl}>2$ (Fig. 2).
Table I. Descriptions of pituitary adenoma samples.

\begin{tabular}{clc}
\hline Sample ID & $\begin{array}{c}\text { Sample } \\
\text { characteristic }\end{array}$ & $\begin{array}{c}\text { NCBI GEO } \\
\text { accession number }\end{array}$ \\
\hline 1 & Invasive & GSM663750 \\
2 & Invasive & GSM663753 \\
3 & Invasive & GSM663754 \\
4 & Invasive & GSM663755 \\
5 & Invasive & GSM663756 \\
6 & Invasive & GSM663757 \\
7 & Invasive & GSM663758 \\
8 & Invasive & GSM96622 \\
9 & Non-invasive & GSM663745 \\
10 & Non-invasive & GSM663747 \\
11 & Non-invasive & GSM663748 \\
12 & Non-invasive & GSM663749 \\
13 & Non-invasive & GSM663751 \\
14 & Non-invasive & GSM663752 \\
15 & Non-invasive & GSM96623 \\
16 & Non-invasive &
\end{tabular}

NCBI GEO, NCBI gene expression omnibus (http://www.ncbi.nlm. nih.gov/geo/).

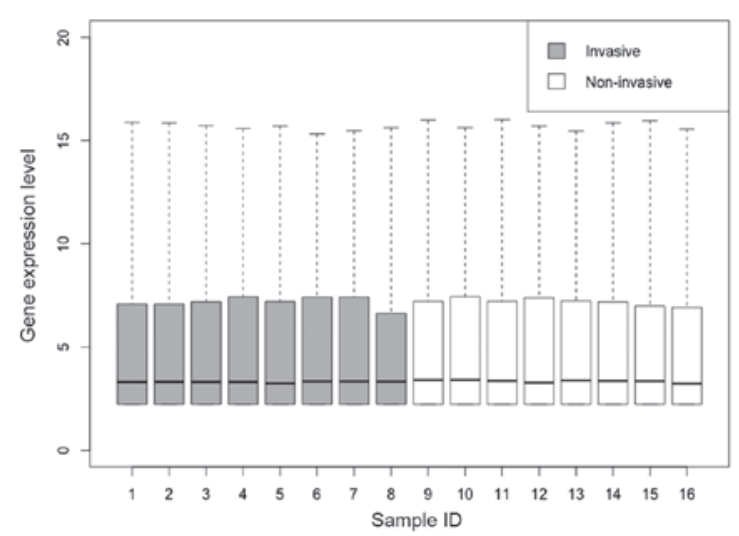

Figure 1. Gene expression data following background correction and normalization. The grey box represents the eight invasive samples and the white box represents eight non-invasive samples from pituitary adenomas. The black line in each box indicates the median of data, and its distribution is able to determine the standardization degree of the data. When the black lines are located at approximately the same level, this indicates an acceptable degree of data pre-processing and normalization.

Gene ontology analysis of differentially expressed genes. As shown in Table II and Fig. 3, the functional gene groups demonstrating the most significant representation in the selected set of differentially expressed genes appear under the biological process ontology and map to cellular biopolymer metabolic process, cellular macromolecule metabolic process, macromolecule metabolic process, primary metabolic process, cellular metabolic process and metabolic process. Functional categories significantly represented under the cellular component and molecular function ontologies include genes involved in the cytoplasm, 
Table II. Ranked list of KEGG pathways impacted in invasive pituitary adenomas.

\begin{tabular}{llcr}
\hline Rank & \multicolumn{1}{c}{ Pathway name } & Impact factor & P-value \\
\hline 1 & Leukocyte transendothelial migration & 188.308 & $3.13 \mathrm{E}-80$ \\
2 & Cell adhesion molecules & 143.574 & $6.41 \mathrm{E}-61$ \\
3 & Adherens junction & 15.326 & $3.60 \mathrm{E}-06$ \\
4 & Circadian rhythm & 11.094 & $1.84 \mathrm{E}-04$ \\
5 & Phosphatidylinositol signalling system & 7.01 & $7.23 \mathrm{E}-03$ \\
\hline
\end{tabular}

KEGG, Kyoto encyclopedia of genes and genomes (http://www.kegg.jp/kegg/pathway.html).
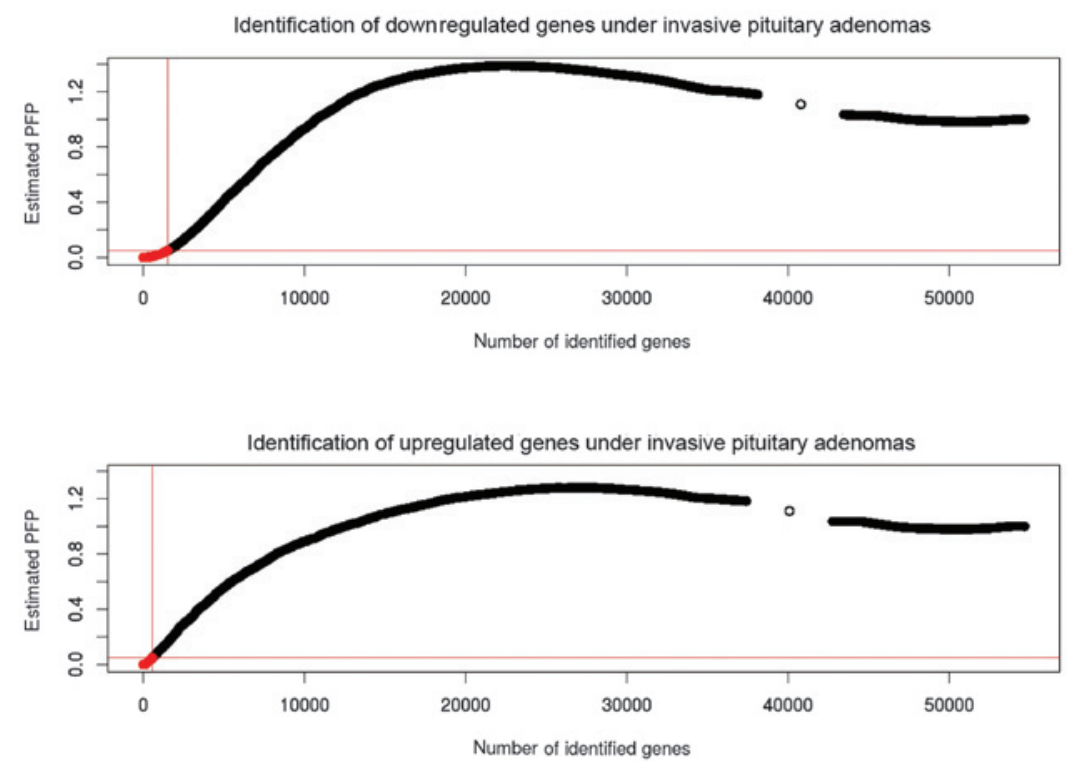

Figure 2. Scatter plot of estimated pfp value versus number of identified differentially expressed genes. Genes within the specified threshold value (pfp $<0.05$ ) range are plotted in red. pfp, false positive prediction.

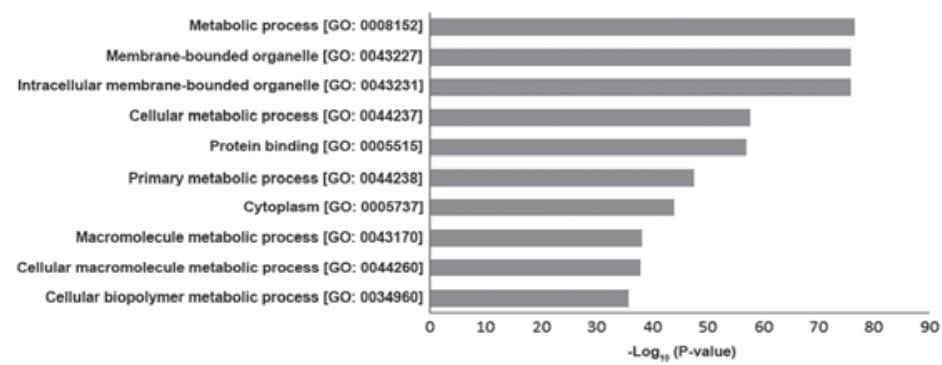

Figure 3. Top ten significant GO terms associated with differentially expressed genes. GO, gene ontology.

protein binding, intracellular membrane-bound organelles and membrane-bound organelles.

Pathway impact analysis. To translate the list of differentially expressed genes into an understanding of the underlying biological phenomena, the web-based software, Pathway-Express, was used to conduct the pathway impact analysis. This analysis included classical statistics and considerations on the type and position of each gene in the specified pathways. As a result, the pathway impact analysis produced more biologically meaningful results than other existing techniques. In the present study, the pathway impact analysis revealed five pathways, which had a significant effect in invasive pituitary adenomas (Table II). These pathways include leukocyte transendothelial migration, cell adhesion molecules (CAMs), adherens junction, circadian rhythm and the phosphatidylinositol signalling system.

Invasion-associated gene identification. As shown in Table II, there are two pathways with significantly higher impact factors, leukocyte transendothelial migration (Fig. 4) and CAMs (Fig. 5). The genes, which were differentially 


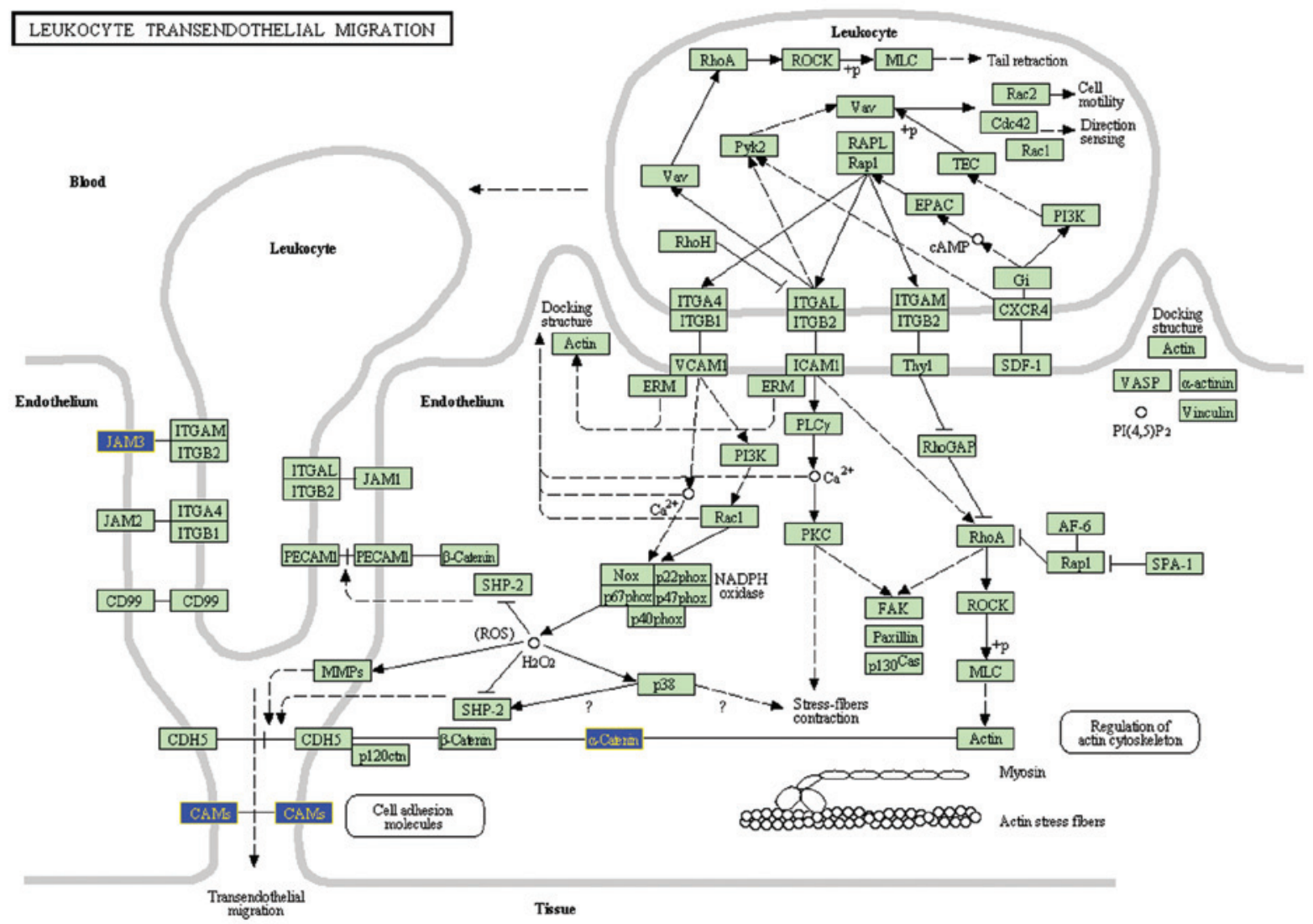

Figure 4. Leukocyte transendothelial migration pathway. Blue boxes indicate the downregulated genes from invasive pituitary adenoma samples.
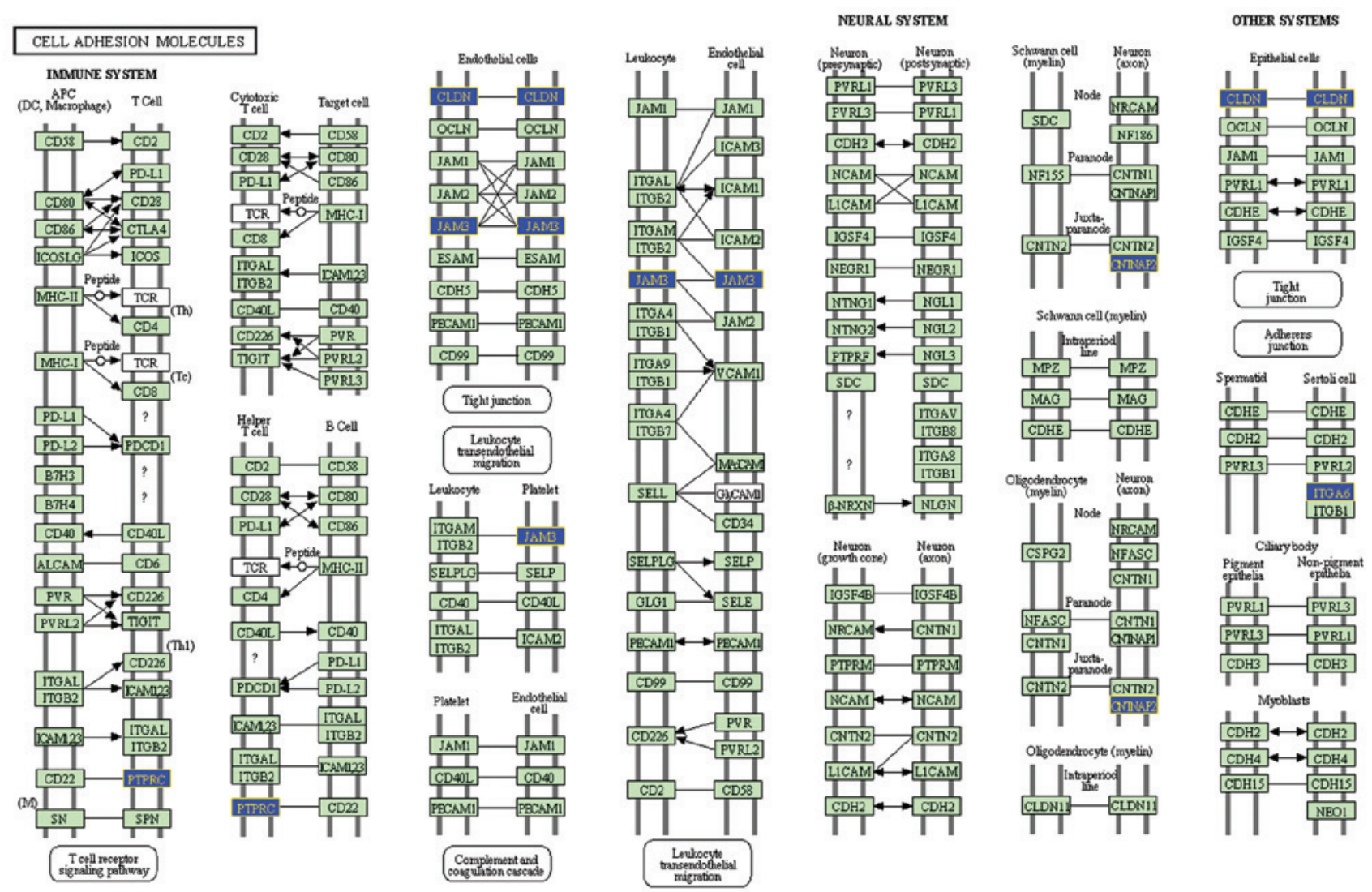

Figure 5. Cell adhesion molecules, Blue boxes indicate the downregulated genes from invasive pituitary adenoma samples. 
expressed in the invasive pituitary adenomas were identified as invasion-associated genes. These genes include claudin 7 (CLDN7), contactin associated protein-like 2 (CNTNAP2), integrin, $\alpha 6$ (ITGA6), junctional adhesion molecule 3 (JAM3), protein tyrosine phosphatase, receptor type, C (PTPRC) and catenin (cadherin-associated protein), $\alpha 1102$ kDa (CTNNA1).

\section{Discussion}

In the present study, the raw intensity files of microarray experiments were retrieved from the NCBI GEO databases. Following pre-processing, RandProd was used to identify differentially expressed genes in invasive pituitary adenomas. Subsequently, Pathway-Express was used to conduct pathway impact analysis. Compared with other gene set enrichment techniques, this approach took consideration of important biological aspects, including the magnitude of each gene's change in expression and their positions in the specified pathways. This provides a greater level of pathway-specific biological analysis than other existing techniques, which render the results more meaningful. The pathway impact analysis revealed two important pathways, leukocyte transendothelial migration and CAMs.

CAMs are important in all aspects of cell growth, cell migration and cell differentiation in vertebrate cells (12). They have been implicated in numerous cellular functions, including signal transduction, cellular communication and recognition, embryogenesis, inflammatory and immune responses, and apoptosis (12). Dysregulation of CAMs is often associated with carcinogenesis (13), particularly tumour invasion. For instance, Cadherin genes are members of the CAMs and are considered to be tumour suppressor genes (14). A member of the Cadherin family, E-cadherin, mediates cell-cell contacts and acts as an important suppressor of epithelial tumour cell invasiveness and metastasis (15). In another study, E-cadherin was observed to be deregulated in the poorly differentiated human squamous cell carcinomas of the head and neck and its expression was inversely correlated with lymph node metastasis (16). In addition to the identification that the E-cadherin-catenin complex is vital in epithelial cell-cell adhesion and in the maintenance of tissue architecture, the expression of the complex was found to be inversely correlated with the invasion of tumour cells (17). These data may support the findings of the present study, which demonstrated that the gene CTNNA1, termed $\alpha$-catenin, was identified as one of the invasion-associated genes in pituitary adenomas.

CLDN7, CNTNAP2, ITGA6, JAM3, PTPRC and CTNNA1 have been identified as invasion-associated genes in pituitary adenomas. In previous studies, it has been reported that reduced expression of CLDN7 correlated with tumour invasion in oesophageal squamous cell carcinoma, colorectal cancer, lung cancer and oral squamous cell carcinoma (18-22). Silencing of ITGA6 was able to significantly inhibit cell migration of head and neck squamous cell carcinoma cells (23). ITGA6 may serve as a potential therapeutic target in oesophageal squamous cell carcinoma (24). In gliomas, the interaction between JAM2 and JAM3 activates the SRC proto-oncogene, which is a central upstream molecule in the pathways that regulated cell migration and invasion (25). The gene JAM3 is also important in the adhesion of cancer cells to extracellular matrices and the subsequent invasion in HT1080 human fibrosarcoma cells (26). Therefore, these six invasion-associated genes identified in the present study may serve as novel diagnostic or therapeutic biomarkers in pituitary adenomas. However, further studies are required.

In conclusion, pathway impact analysis was used to identify an invasion-associated molecular signature of six genes, CLDN7, CNTNAP2, ITGA6, JAM3, PTPRC and CTNNA1. These genes were significantly deregulated in invasive pituitary adenomas and may serve as potential diagnostic or therapeutic biomarkers in the treatment of invasive pituitary adenomas. However, further studies are required to validate the present findings.

\section{References}

1. Oruçkaptan HH, Senmevsim O, Ozcan OE and Ozgen T: Pituitary adenomas: results of 684 surgically treated patients and review of the literature. Surg Neurol 53: 211-219, 2000.

2. Scheithauer BW, Kurtkaya-Yapicier O, Kovacs KT, Young WF Jr and Lloyd RV: Pituitary carcinoma: a clinicopathological review. Neurosurgery 56: 1066-1074, 2005.

3. Thapar K, Scheithauer BW, Kovacs K, Pernicone PJ and Laws ER Jr: p53 expression in pituitary adenomas and carcinomas: correlation with invasiveness and tumor growth fractions. Neurosurgery 38: 765-770, 1996.

4. Ezzat S, Zheng L and Asa SL: Pituitary tumor-derived fibroblast growth factor receptor 4 isoform disrupts neural cell-adhesion molecule/ $\mathrm{N}$-cadherin signaling to diminish cell adhesiveness: a mechanism underlying pituitary neoplasia. Mol Endocrinol 18: 2543-2552, 2004.

5. Kawamoto H, Kawamoto K, Mizoue T, Uozumi T, Arita K and Kurisu K: Matrix metalloproteinase-9 secretion by human pituitary adenomas detected by cell immunoblot analysis. Acta Neurochir (Wien) 138: 1442-1448, 1996.

6. Gadelha MR, Trivellin G, Hernández Ramírez LC and Korbonits M: Genetics of pituitary adenomas. Front Horm Res 41: 111-140, 2013.

7. Barrett T, Wilhite SE, Ledoux P, et al: NCBI GEO: archive for functional genomics data sets - update. Nucleic Acids Res 41: D991-D995, 2013.

8. Rustici G, Kolesnikov N, Brandizi M, et al: ArrayExpress update-trends in database growth and links to data analysis tools. Nucleic Acids Res 41: D987-D990, 2013.

9. Gautier L, Cope L, Bolstad BM and Irizarry RA: affy-analysis of Affymetrix GeneChip data at the probe level. Bioinformatics 20: 307-315, 2004.

10. Hong F, Breitling R, McEntee CW, Wittner BS, Nemhauser JL and Chory J: RankProd: a bioconductor package for detecting differentially expressed genes in meta-analysis. Bioinformatics 22: 2825-2827, 2006.

11. Tarca AL, Draghici S, Khatri P, et al: A novel signaling pathway impact analysis. Bioinformatics 25: 75-82, 2009.

12. Cohen MB, Griebling TL, Ahaghotu CA, Rokhlin OW and Ross JS: Cellular adhesion molecules in urologic malignancies. Am J Clin Pathol 107: 56-63, 1997.

13. Okegawa T, Pong RC, Li Y and Hsieh JT: The role of cell adhesion molecule in cancer progression and its application in cancer therapy. Acta Biochim Pol 51: 445-457, 2004.

14. Okegawa T, Li Y, Pong RC and Hsieh JT: Cell adhesion proteins as tumor suppressors. J Urol 167: 1836-1843, 2002.

15. Birchmeier $\mathrm{W}$ and Behrens $\mathrm{J}$ : Cadherin expression in carcinomas: role in the formation of cell junctions and the prevention of invasiveness. Biochim Biophys Acta 1198: 11-26, 1994.

16. Behrens J: The role of cell adhesion molecules in cancer invasion and metastasis. Breast Cancer Res Treat 24: 175-184, 1993.

17. Wijnhoven BP, Dinjens WN and Pignatelli $M$ : E-cadherin-catenin cell-cell adhesion complex and human cancer. Br J Surg 87: 992-1005, 2000.

18. Usami Y, Chiba H, Nakayama F, et al: Reduced expression of claudin-7 correlates with invasion and metastasis in squamous cell carcinoma of the esophagus. Hum Pathol 37: $569-577,2006$. 
19. Lioni M, Brafford P, Andl C, et al: Dysregulation of claudin-7 leads to loss of E-cadherin expression and the increased invasion of esophageal squamous cell carcinoma cells. Am J Pathol 170: 709-721, 2007.

20. Oshima T,Kunisaki C, Yoshihara K, et al: Reduced expression of the claudin-7 gene correlates with venous invasion and liver metastasis in colorectal cancer. Oncol Rep 19: 953-959, 2008.

21. Lu Z, Ding L, Hong H, Hoggard J, Lu Q and Chen YH: Claudin-7 inhibits human lung cancer cell migration and invasion through ERK/MAPK signaling pathway. Exp Cell Res 317: 1935-1946, 2011.

22. Yoshizawa K, Nozaki S, Kato A, et al: Loss of claudin-7 is a negative prognostic factor for invasion and metastasis in oral squamous cell carcinoma. Oncol Rep 29: 445-450, 2013.
23. Kinoshita T, Nohata N, Hanazawa T, et al: Tumour-suppressive microRNA-29 s inhibit cancer cell migration and invasion by targeting laminin-integrin signalling in head and neck squamous cell carcinoma. Br J Cancer 109: 2636-2645, 2013.

24. Kwon J, Lee TS, Lee HW, et al: Integrin alpha 6: a novel therapeutic target in esophageal squamous cell carcinoma. Int J Oncol 43: 1523-1530, 2013.

25. Tenan M, Aurrand-Lions M, Widmer V, et al: Cooperative expression of junctional adhesion molecule-C and -B supports growth and invasion of glioma. Glia 58: 524-537, 2010.

26. Fuse $\mathrm{C}$, Ishida $\mathrm{Y}$, Hikita $\mathrm{T}$, Asai $\mathrm{T}$ and Oku N: Junctional adhesion molecule-C promotes metastatic potential of HT1080 human fibrosarcoma. J Biol Chem 282: 8276-8283, 2007. 